\title{
EL NUEVO ENIGMA DE LA INDUCCIÓN Y LOS TÉRMINOS DE CLASE NATURAL
}

\author{
Ignacio Ávila CaÑamares \\ Departamento de Filosofía \\ Universidad Nacional de Colombia \\ ignacio_avila@hotmail.com
}

RESUMEN: En este ensayo intento mostrar el estrecho vínculo entre el asunto de la fijación de la extensión de los términos de clase natural y el problema de la proyectabilidad puesto de relieve por Goodman con su nuevo enigma de la inducción. Por un lado argumento que el nuevo enigma de la inducción pone de manifiesto la presencia de un elemento fregeano en la teoría de la referencia directa de Putnam y, por el otro, señalo la necesidad de que una respuesta realista a dicho enigma se articule con una tesis epistemológica de cómo conocemos las presuntas propiedades intrínsecas de los objetos.

PALABRAS CLAVE: realismo, referencia, proyectabilidad, muestras

SUMMARY: This paper intends to show the tight link between the question of fixation of extension of natural kind terms and the problem of projectibility pointed out by Goodman's new riddle of induction. I will argue that, on the one hand, the new riddle of induction enables us to show that Putnam's theory of reference has a Fregean element. On the other, I will point out that a realist answer to the new riddle must be articulated with an epistemological thesis about our knowledge of pretended intrinsic properties of objects.

KEY WORDS: realism, reference, projectibility, samples

El debate filosófico en torno a las clases naturales atraviesa varias discusiones y problemas pertenecientes a diferentes ámbitos de la reflexión filosófica. Las clases naturales aparecen involucradas en los debates ontológicos acerca del estatus de los universales y las leyes científicas, en las controversias epistemológicas sobre la inducción y la proyectabilidad, en los problemas semánticos tejidos alrededor de los términos de clase natural, e incluso en cuestiones propias de la filosofía de la ciencia, tales como el debate en torno al relativismo o la manera misma como ha de entenderse la actividad científica. Si bien es de suponer que 
todos estos problemas se encuentran relacionados entre sí, parte de la dificultad radica en establecer el sentido preciso de tales relaciones. Entre la gran cantidad de discusiones a las que han dado lugar las clases naturales, ocupan un lugar destacado el debate en torno al así llamado nuevo enigma de la inducción de Goodman y la discusión en torno a la denominada teoría directa de la referencia de Kripke y Putnam.

A primera vista, puede parecer que no existe conexión alguna entre el nuevo enigma de la inducción y las teorías de la referencia directa de Kripke y Putnam. Sin embargo, la intuición que alimenta este ensayo es que el problema de la proyectabilidad señalado por el nuevo enigma de la inducción está íntimamente ligado al asunto de la fijación de la extensión de los términos de clase natural y, en este sentido, un estudio conjunto de ambos temas permite sacar a la luz ciertas dificultades inherentes a cada uno de ellos. En particular, intentaré argumentar, por un lado, que no es suficiente apelar a una teoría de la referencia directa para proporcionar una respuesta al nuevo enigma de la inducción que satisfaga a alguien con fuertes intuiciones realistas y, por el otro, que este enigma permite poner de relieve ciertas dificultades propias de las teorías de la referencia directa. Asimismo, sugeriré que la respuesta de Goodman al nuevo enigma de la inducción no es suficiente por sí misma para sustentar su bien conocido irrealismo, ni implica necesariamente una concepción nominalista de las clases naturales como la que él defiende.

El desarrollo de este ensayo será, a grandes rasgos, el siguiente: en la primera sección presentaré el nuevo enigma de la inducción y cómo Goodman intenta resolverlo. Esto nos permitirá mostrar por qué esta respuesta resulta insatisfactoria para alguien que mantiene intuiciones realistas y antirrelativistas, y nos permitirá esbozar una respuesta acorde con tales intuiciones. En la segunda sección expondré la teoría de la referencia directa desarrollada por H. Putnam en "El Significado de "Significado" y trataré de mostrar la forma en que ésta podría apoyar una eventual respuesta al nuevo enigma de la inducción diferente de la que ofrece Goodman. Luego, en la tercera sección, discutiré esta respuesta, lo cual nos ayudará a sacar a la luz un "elemento fregeano" oculto en la teoría de la referencia directa 
de Putnam que puede diluir la respuesta realista y antirrelativista al nuevo enigma de la inducción en una respuesta muy similar a la de Goodman. Finalmente, en la cuarta sección, exploraré rápidamente algunas de las perspectivas que deja abiertas el debate en torno al nuevo enigma de la inducción y las teorías directas de la referencia cuando se las considera desde una óptica común.

\section{El nuevo enigma de la inducción}

Goodman formula el nuevo enigma de la inducción de la siguiente manera (cfr. Goodman 1983, cap. 3): Supongamos que todas las esmeraldas observadas antes de cierto tiempo futuro $t$ han sido verdes. Esta regularidad nos lleva a inferir que todas las esmeraldas observadas y no observadas son verdes. De hecho, las esmeraldas examinadas hasta ahora parecen confirmar de sobra esta inferencia inductiva. Sin embargo, el asunto no es tan fácil como parece a primera vista. Goodman nos invita a que consideremos el predicado "verdul" definido como sigue: un objeto es verdul si y sólo si ha sido examinado antes de un tiempo futuro específico $t$ y es verde o no ha sido examinado antes de $t$ y es azul. De acuerdo con esto, es indiscutible que las esmeraldas examinadas antes de $t$ son verdules $\mathrm{y}$, en este sentido, cualquier evidencia a favor de que las esmeraldas futuras son verdes es también una evidencia a favor de que son verdules y, a fortiori, de que son azules. Parece entonces tan lícito concluir, a partir de la evidencia recolectada hasta ahora, que todas las esmeraldas son verdes como concluir que son verdules. Sin embargo, no estamos dispuestos a aceptar la hipótesis que afirma la verdulez de las esmeraldas. Más bien, nos inclinamos a suponer que sólo la hipótesis de que todas las esmeraldas son verdes es válida. El problema es que no hemos dado ninguna razón para preferir esta hipótesis a su rival. El nuevo enigma de la inducción consiste entonces en explicar por qué preferimos proyectar hacia el futuro unas hipótesis en vez de otras o por qué consideramos que unas inferencias inductivas son válidas y otras no, si todas ellas son compatibles con la totalidad de la evidencia recolectada hasta ahora. 
La reacción inmediata a este problema es alegar que el predicado "verdul" no es legítimo y como tal no puede formar parte de ninguna inferencia inductiva válida. El problema está justamente en saber por qué no lo es. La respuesta más natural es sugerir que un predicado legítimo — contrario a lo que ocurre con "verdul" - no puede ser disyuntivo o no puede contener una referencia a un punto particular del tiempo. Sin embargo, esta opción es inadecuada. Goodman señala que es cierto que si tomamos como primitivos los predicados "verde" y "azul", podemos definir "verdul" o "azurde" (azul y examinado antes de $t$, o verde después de $t$ ) en términos de ellos y una referencia a un punto específico en el tiempo. Pero es igualmente cierto que si tomamos como primitivos los predicados "verdul" y "azurde", podemos definir de la misma forma "verde" y "azul". Desde este punto de vista, un objeto es verde si y sólo si es verdul y examinado antes de $t$, o azurde después de $t$ y, del mismo modo, algo es azul si y sólo si es azurde y examinado antes de $t$, o verdul después de $t$. De este modo, Goodman concluye que el intento de rechazar predicados como "verdul" imputándoles su carácter disyuntivo y su dependencia con respecto a instantes de tiempo específicos está condenado al fracaso. Sobre la misma base, también podríamos rechazar predicados como "verde" que nos resultan perfectamente naturales.

De acuerdo con esto, Goodman sostiene que el nuevo enigma de la inducción revela dos aspectos importantes de los predicados del lenguaje y de la lógica de la inferencia inductiva. Por un lado, pone de manifiesto que los predicados no poseen propiedades esenciales independientes de la forma en que los usemos. Los predicados no son per se disyuntivos o no disyuntivos, legítimos o ilegítimos, normales o anormales. Un predicado como "verdul" puede parecer disyuntivo o anormal si tomamos como primitivos "verde" y "azul", pero, como hemos visto, la inversa es igualmente cierta. Por el otro, el nuevo enigma de la inducción revela que la lógica inductiva no es puramente formal. Desde un punto de vista lógico, tanto la hipótesis de que todas las esmeraldas son verdes como la de que todas las esmeraldas son verdules comparten la misma forma lógica. Por consiguiente, contrario a lo que 
ocurre con la deducción, la sola forma lógica no nos permite determinar la validez de una inferencia inductiva. Así, si queremos determinar la validez de una inferencia inductiva, es necesario tener en cuenta también el tipo de predicados que utilizamos en ella. Entonces, el reto del nuevo enigma de la inducción consiste en obligarnos a ofrecer un criterio que nos permita discriminar, entre todas las inferencias inductivas que comparten la misma forma lógica, aquellas que son válidas de aquellas que no lo son. Y dado que la discusión en torno a la validez de las inferencias inductivas debe pasar por una consideración acerca del tipo de predicados aceptables en tales inferencias, el nuevo enigma de la inducción puede entenderse como el problema de establecer pautas que permitan determinar cuáles predicados son proyectables en una inferencia inductiva y cuáles no. La idea - claro está- es que nuestra respuesta asegure la proyectabilidad de "verde", pero excluya la de "verdul".

La respuesta general de Goodman al nuevo enigma de la inducción descansa en la noción de atrincheramiento (entrenchment). Goodman no sugiere que su respuesta sea la única posible al problema tan sólo enfatiza que cualquier respuesta que se nos ocurra debe estar en concordancia con nuestras prácticas inductivas cotidianas y científicas. Goodman sostiene que los predicados proyectables en una inducción son aquellos que están mejor atrincherados, es decir, que se han usado con más frecuencia en predicciones pasadas. La proyectabilidad de los predicados dependerá entonces de su historial de uso al interior de un lenguaje, de la frecuencia con que los hemos proyectado en el pasado; en una palabra, de su currículum. La idea de Goodman es que así como esperamos que las regularidades observadas en el pasado se mantengan en el futuro, así también proyectamos hacia el futuro los predicados que hemos utilizado con éxito en el pasado. De acuerdo con esto, queda claro por qué consideramos que la hipótesis de que todas las esmeraldas son verdes es válida y rechazamos la hipótesis de que sean verdules. El predicado "verde" está mejor atrincherado en nuestro lenguaje que el predicado "verdul"; ha sido usado exitosamente en muchas inducciones en el pasado. Si nuestro lenguaje fuera diferente y "verdul" estuviera mejor atrincherado, la inferencia de que 
todas las esmeraldas son verdules sería válida. Así, la validez de una inferencia inductiva dependerá no sólo de su forma lógica y su concordancia con la evidencia disponible, sino también del lenguaje con el que registramos dichas observaciones. Al igual que en el caso de los predicados, aquí tampoco hay inferencias inductivas válidas o inválidas per se.

La respuesta de Goodman al nuevo enigma de la inducción se articula con varios sectores de su filosofía, tales como su aceptación del relativismo y el irrealismo. Si la proyectabilidad de los predicados depende de su grado de atrincheramiento en un lenguaje y de la forma como hayamos dividido el mobiliario del mundo, entonces en un lenguaje constituido por excéntricos predicados goodmanescos como "verdul" cualquier inferencia inductiva basada en nuestros predicados actuales sería inválida. Si nuestro lenguaje estuviera constituido por predicados goodmanescos, no estaríamos dispuestos a considerar que los tigres, las esmeraldas o las cosas verdes sean clases naturales. En su lugar, habitaríamos un mundo en el cual los tigrefantes, los esmefiros y las cosas verdules constituirían clases naturales genuinas. De este modo, la respuesta de Goodman al nuevo enigma de la inducción se complementa con su bien conocido irrealismo. A grandes rasgos, él considera que la idea de que existe un único mundo independiente de nosotros y organizado de antemano de una única forma carece de sentido y, más bien, sugiere que existe toda una pluralidad de mundos actuales indistinguibles de las diferentes versiones de mundo que construimos (cfr. Goodman 1990, cap. 1 y Goodman 1995, cap. 2, por ejemplo).

Según esta concepción irrealista, las así llamadas clases naturales adquieren un estatus especial. Ellas no existen dadas de antemano en un mundo independiente y a la espera de que las descubramos, sino que más bien forman parte de nuestras versiones de mundo. Hacia el final de Fact, Fiction and Forecast, Goodman escribe:

Nuestro tratamiento de la proyectabilidad resulta promisorio en otras direcciones; nos puede proporcionar una manera de distinguir clases "genuinas" de clases meramente "artificiales" y, de este modo, nos capacita para interpretar los enunciados comunes 
que afirman que ciertas cosas son o no son de la misma clase, o que ciertas cosas son más similares que otras. Pues seguramente el atrincheramiento de las clases proporciona alguna medida de su carácter genuino (genuineness) como clases. (Goodman 1983, p. 123)

Pero si la distinción entre clases genuinas y no genuinas descansa en el atrincheramiento de predicados, entonces lenguajes con predicados atrincherados distintos se comprometerán con diferentes clases, todas ellas genuinas intralingüísticamente. En este sentido, Goodman se opone a la idea de que hay clases genuinas o naturales que en sí mismas constituyen la estructura última del mundo y más bien considera que, estrictamente hablando, sólo existen clases artificiales construidas de acuerdo con los diferentes lenguajes.

Es evidente que la respuesta de Goodman al nuevo enigma de la inducción resultará insatisfactoria para un defensor del realismo robusto. La tesis del realista robusto es, en su forma más general, que existe un mundo independiente de nosotros que posee en sí mismo una estructura propia. ${ }^{1}$ Por lo tanto, el realista robusto se opondrá a la tesis irrealista de Goodman según la cual todas las clases son artificiales y a la tesis de que la distinción entre clases genuinas y no genuinas depende, en última instancia, del lenguaje. En opinión del realista robusto, el mundo está conformado en sí mismo por clases naturales y, en este sentido, la distinción entre clases genuinas y no genuinas no puede ser una función del lenguaje. Para él, las clases genuinas serán simplemente las clases naturales en las que está estructurado el mundo, independientemente del lenguaje que poseamos.

Desde este punto de vista, el realista robusto se sentirá insatisfecho con la respuesta de Goodman al nuevo enigma de la inducción y, en su lugar, buscará una respuesta acorde con sus intuiciones. Para ello, mantendrá la idea de que un predicado sólo es proyectable si tiene como extensión una clase genuina sin renunciar a la idea realista de que tales clases conforman la estructura última del mundo. Con esto, el realista robusto

${ }^{1}$ Para una caracterización más detallada de la tesis del realismo en general y del realismo robusto en particular, véase Devitt 1991, cap. I. 
podrá proporcionar su propia respuesta al nuevo enigma de la inducción en los siguientes términos: si se concede que sólo los predicados proyectables pueden formar parte de una inferencia inductiva válida y luego — dado el compromiso con el realismose argumenta que sólo son proyectables aquellos predicados que tienen como extensión una de las clases naturales que conforman la estructura del mundo, entonces la única conclusión que cabe es que la validez de una inferencia inductiva estará en última instancia dictada por el mundo. Con esta conclusión, el realista robusto podrá rechazar la propuesta goodmaniana que hace depender la validez de una inferencia inductiva de las prácticas lingüísticas vigentes al interior de una comunidad. La pregunta, claro está, es si puede construirse un argumento que permita alcanzar la conclusión que el realista antirrelativista se propone.

En principio es posible ensayar una propuesta en esta dirección. De acuerdo con ella, Goodman estaría en lo correcto al sostener que los predicados proyectables deben tener como extensión una clase genuina. Su error habría sido suponer que lo que cuenta como una clase genuina depende necesariamente del lenguaje y, a partir de allí, apelar al atrincheramiento como criterio de validez inductiva. El núcleo de esta propuesta radicaría en mostrar que, contrario a lo que sostiene Goodman, la extensión de los predicados proyectables no está determinada por nuestros lenguajes o sistemas de creencias, sino que está fijada por el mundo. Esto permitiría resolver el nuevo enigma de la inducción en términos realistas, pues sólo aquellos predicados cuya extensión esté dada por el mundo podrían tomar parte en inferencias inductivas válidas. Así, la razón por la cual la hipótesis que sostiene que todas las esmeraldas son verdes es válida no radicaría en que el predicado "verde" esté bien atrincherado en nuestro lenguaje, sino en que - a diferencia de "verdul" - su extensión es una de las clases naturales que conforman el mundo. ${ }^{2}$ Obviamente, la viabilidad de esta propuesta dependerá de que contemos con una teoría de la referencia que

2 Puede encontrarse un intento de resolver el nuevo enigma de la inducción en estos términos en Lewis 1997. 
permita mostrar que la extensión de un término está fijada por el mundo mismo. Precisamente, Putnam proporciona dicha teoría.

\section{La teoría de la referencia directa de Putnam}

De acuerdo con Putnam, las teorías tradicionales de la referencia descansan en dos supuestos que combinados resultan inconsistentes. Tales supuestos son: (i) Conocer el significado de un término no es sino estar en cierto estado psicológico, y (ii) el significado de un término determina su extensión. Putnam considera que la conjunción de estos dos supuestos conlleva a la teoría de que los estados psicológicos determinan la extensión de nuestros términos. La estrategia de Putnam consistirá entonces en mostrar que esta idea está equivocada. Él argumentará que el supuesto (i) es falso y el supuesto (ii) sólo puede aceptarse en una versión muy debilitada. Por consiguiente, Putnam sostendrá que - contrario a lo que sostienen las teorías tradicionales de la referencia- la extensión de un término no está determinada por las creencias del hablante, sino que viene dictada por la comunidad de expertos y el mundo.

Gran parte de la argumentación de Putnam está sustentada en contraejemplos dirigidos a mostrar que - contrario a los supuestos (i) y (ii) — dos hablantes o dos comunidades lingüísticas pueden tener los mismos estados psicológicos o compartir creencias idénticas y, sin embargo, sus términos pueden diferir en su extensión. El contraejemplo más relevante es el conocido caso de la Tierra Gemela. Supongamos que en una galaxia lejana existe una Tierra Gemela que es casi idéntica a nuestro planeta. Allí también hablan español, poseen una división geográfica como la nuestra y sus avances científicos corren paralelos a los nuestros. La única diferencia existente entre ambas tierras es que, en la Tierra Gemela, el líquido que los hispanoparlantes llaman "agua" no es $\mathrm{H}_{2} \mathrm{O}$ sino XYZ. Sin embargo, esta diferencia no es detectable a menos que se cuente con las herramientas de la química daltoniana. Superficialmente $\mathrm{H}_{2} \mathrm{O}$ y XYZ son indistinguibles. Ambos quitan la sed, son incoloros e insípidos, etc. Si ahora retrocedemos hasta 1750 cuando no se conocía en ninguna Tierra la química daltoniana, entonces, ex hypothesi, 
ningún habitante de ambos planetas podría distinguir entre los dos líquidos. Antes bien, podemos suponer que los habitantes de las dos tierras asociarían creencias idénticas al término "agua". Todos ellos considerarían que el agua es un líquido incoloro e insaboro que calma la sed, se encuentra en los lagos y los ríos, etc. Sin embargo - y éste es el punto clave de Putnam-, la extensión del término "agua" en ambas tierras es diferente. En la Tierra, la extensión de "agua" es $\mathrm{H}_{2} \mathrm{O}$, mientras que en la Tierra Gemela su extensión es XYZ. Pero si es así, entonces los supuestos (i) y (ii) entendidos a la manera tradicional deben ser abandonados. Justamente, estamos ante un caso en el cual los hablantes de dos comunidades lingüísticas poseen creencias idénticas $y$, sin embargo, la extensión de sus términos es diferente.

Ahora bien, si la extensión de los términos de un lenguaje no está determinada por las creencias de los usuarios del lenguaje, entonces habrá de estar fijada por el mundo. De hecho, Putnam argumenta que la extensión de "agua" y los demás términos de clase natural se fija mediante la siguiente definición ostensiva:

Supongamos que señalo un vaso de agua y digo "este líquido se llama agua" (o bien: "a esto se le llama agua", suponiendo que el marcador "líquido" se da como obvio). Mi "definición ostensiva" de agua tiene el siguiente presupuesto empírico: que la porción del líquido que estoy señalando se encuentra en una cierta relación de mismidad (por ejemplo, $x$ es el mismo líquido que $y$, o $x$ es lo mismo que y) con la mayor parte de la sustancia a la que yo y otros hablantes de mi comunidad lingüística hemos llamado "agua" en otras ocasiones. [...] La condición necesaria y suficiente que debe reunir algo para ser agua es la de hallarse en la relación mismo ${ }_{L}$ con la sustancia del vaso; pero ésta es la condición necesaria y suficiente sólo si el presupuesto empírico se cumple. (Putnam 1991, p. 142)

Así pues, los términos de clase natural poseen un elemento indexical oculto que permite fijar su extensión: esto (y se lo señala) es agua si y sólo si está en la relación mismo $L$ que lo que llamamos "agua" y - la inversa también vale- agua es todo aquello que está en la relación mismo $L$ con esta sustancia (y se la señala). Pero, de acuerdo con Putnam, decir que los términos 
de clase natural poseen un indexical oculto no sólo equivale a afirmar que la fijación de su extensión no está determinada por el sistema de creencias de los hablantes, sino que - en su opinión - también pone de manifiesto que tales términos son designadores rígidos. Si agua es todo aquello que está en la relación mismo $L$ con esta muestra de agua, entonces nuestra definición ostensiva de "agua" implicará que con dicho término se designará al mismo tipo de sustancia en todos los mundos posibles. Así, Putnam concluye:

Nuestra teoría puede resumirse diciendo que las palabras como "agua" tienen un elemento indicador (indexical) oculto: el "agua" es una sustancia que guarda con el agua de por aquí una cierta relación de similaridad. En un tiempo o en un lugar distintos, o incluso en otro mundo posible, el agua, si es que ha de ser agua, ha de estar con nuestra agua en la relación $\operatorname{mismo}_{L}$. Así pues, la teoría de que (1) las palabras tienen "intensiones", que son algo parecido a los conceptos vinculados a las palabras de los hablantes; y que (2) las intensiones determinan la extensión, no puede ser verdadera en lo que toca a las palabras que designan clases naturales, como "agua". (Putnam 1991, p. 152)

No obstante, Putnam reconoce que el hecho de que nuestros conceptos y creencias no determinen la extensión de un término no quiere decir que el significado, entendido en un sentido amplio, se identifique con la extensión. Más bien, Putnam entiende el significado de un término como un vector construido de la siguiente manera:

$\begin{array}{llll}\begin{array}{l}\text { Marcadores } \\ \text { sintácticos }\end{array} & \begin{array}{l}\text { Marcadores } \\ \text { semánticos }\end{array} & \text { Estereotipo } & \text { Extensión } \\ \begin{array}{l}\text { nombre } \\ \text { de masa; } \\ \text { concreto }\end{array} & \begin{array}{l}\text { clase natural; } \\ \text { líquido }\end{array} & \begin{array}{l}\text { incoloro; } \\ \text { transparente; } \\ \text { insípido; } \\ \text { que calma } \\ \text { la sed; etc. }\end{array} & \begin{array}{l}\mathrm{H}_{2} \mathrm{O} \\ \text { (con o sin } \\ \text { impurezas) }\end{array} \\ & & & \end{array}$

Con respecto a este vector son pertinentes las siguientes aclaraciones: en primer lugar, este vector pone de manifiesto el sentido 
debilitado en el cual cabe decir que el significado de un término determina su extensión. Esto simplemente quiere decir que la extensión forma parte del vector que constituye el significado de un término, lo cual es trivialmente verdadero. Pero de aquí no se sigue que los conceptos o creencias que el hablante liga a un término determinen su extensión. La intensión y la extensión son dos componentes independientes del significado de un término. En segundo lugar, los estereotipos de Putnam son, dicho toscamente, el conjunto de ideas asociadas a un término que exigimos que conozca una persona dentro de nuestra comunidad lingüística para considerarla competente en el manejo de dicho término. Por su parte, los marcadores semánticos son las ideas asociadas a un término que consideramos virtualmente irrevisables. Si consideramos el significado de "tigre", rasgos como el ser de cierto tamaño, tener rayas negras o vivir solitarios en la selva formarán parte de su estereotipo. Sin embargo, el rasgo de ser animal posee un estatus diferente; es un marcador semántico que difícilmente estaríamos dispuestos a abandonar. Fácilmente renunciamos a la idea de que todos los tigres tienen rayas o viven en la selva, pero difícilmente dejaríamos de creer que los tigres son animales. De acuerdo con esto, puede decirse que tanto los estereotipos como los marcadores semánticos constituyen la intensión de un término; lo que ocurre es que no determinan su extensión. Esto permite explicar la situación de los habitantes de las dos Tierras ante $\mathrm{H}_{2} \mathrm{O}$ y XYZ. Lo que ocurría allí era simplemente que sus habitantes asociaban los mismos estereotipos y marcadores semánticos al término "agua", pero — dadas las características de cada Tierra - se referían a líquidos diferentes.

Ahora podemos examinar cómo la teoría de la referencia de Putnam podría apoyar la respuesta del realista robusto al nuevo enigma de la inducción. En la sección anterior sugerí que una respuesta tal tenía que mantener el vínculo entre predicados proyectables y clases naturales, sin abandonar el realismo con respecto a ellas. Ahora parece que la teoría de la referencia de Putnam lleva a feliz término un esfuerzo en esta dirección. Esto es por varias razones. La primera de ellas radica en que la teoría de la referencia directa implica, ipso facto, un compromiso con un realismo robusto. Esto se desprende de la tesis misma de que 
la extensión de un término no está determinada por nuestras creencias particulares sino por el mundo. "Oro", por ejemplo, se referirá a todo aquello que mantenga la relación mismo metal con este pedazo de oro que señalamos, independientemente de cuáles sean nuestras creencias sobre el oro. En este sentido, resultará tan absurdo decir que estipulamos cuál es la extensión de "oro" de acuerdo con nuestras creencias particulares, como decir que construimos dicha clase natural. Idénticos sistemas de creencias no construirán mundos goodmanianos idénticos, ni sistemas diferentes harán mundos diferentes. Los habitantes de las dos Tierras han construido versiones de mundos idénticas (por usar la expresión de Goodman) y, sin embargo, la extensión de sus términos es diferente. Igualmente, podemos imaginar dos versiones de mundo en conflicto, tales que en una de ellas "oro" incluye anillos de oro mientras que en la otra no y, sin embargo, no estaremos en mundos diferentes. Simplemente, los anillos formarán parte de la extensión de "oro" si están en la relación $\operatorname{mismo}_{M}$ con el oro. En ambos casos contamos con un dominio de entidades fijo e independiente de cualquier versión de mundo que fija la extensión de nuestros términos.

En segundo lugar, la teoría de la referencia de Putnam se opone al relativismo que impregna la respuesta de Goodman al nuevo enigma de la inducción. La razón de ello es la misma que la anterior. Si la extensión de los términos de clase natural está fijada por el mundo, entonces no se verá afectada en modo alguno por las transformaciones de nuestras creencias. En este sentido, no habrá inconmensurabilidad entre paradigmas diferentes o, al menos, no en cuanto a la extensión de los términos que emplean.

En dos mil años - escribe Putnam - "oro" no ha cambiado de extensión (o no ha cambiado de modo significativo). Nuestros métodos de verificación se han hecho increíblemente sofisticados. Pero la extensión de Xrisos en el dialecto del griego de Arquímedes es la misma que la extensión de oro en mi dialecto español". (Putnam 1991, p. 153)

Con todo, el beneficio más grande de la teoría de Putnam para quien intente resolver el nuevo enigma de la inducción desde una 
perspectiva realista es que vincula directamente los términos de clase natural con el mundo. Esto no sólo impide un divorcio entre las clases genuinas intralingüísticamente y las clases naturales en las que se estructura realmente el mundo, sino que además permite establecer las pautas que ha de satisfacer una inducción válida y nos alivia de los excéntricos predicados goodmanescos. Desde esta perspectiva, las clases genuinas intralingüísticamente serán las clases naturales en tanto que los términos de clase natural poseen un elemento indexical. De hecho, la idea de clases genuinas separadas del mundo carecerá de sentido: sencillamente no serán clases genuinas. Pero si es así, la validez de una hipótesis dependerá de que contenga términos de clase natural y sólo tales términos serán proyectables. De esta manera, predicados como "verdul" no formarán parte de una inferencia inductiva válida puesto que no son genuinos términos de clase natural.

Para ver este punto de forma más clara, supongamos que definimos ostensivamente "esmeralda" como todo aquello que está en la relación misma piedra preciosa que ésta muestra, donde algo está en la relación misma $P P$ con una esmeralda si posee la estructura molecular ESME. Supongamos, además, que hacemos algo parecido con "zafiro" y la fórmula química del zafiro es FIRO. En este sentido, resulta claro que "esmefiro" (definido como esmeralda observada antes de $t$, o zafiro después) no puede ser un término de clase natural, pues esto implicaría que la fórmula química de un esmefiro ha de ser algo similar a un compuesto entre ESME y FIRO y, de este modo, no sería - como estipula la definición de "esmefiro" - ni una esmeralda antes de $t$ ni un zafiro después. Incluso cuando después de $t$ encontráramos que las piedras que antes eran esmeraldas ahora son zafiros, no diríamos por ello que las esmeraldas son esmefiros. Simplemente diríamos que las esmeraldas han sido reemplazadas por los zafiros. Un caso como éste no es un caso en el cual las hipótesis inductivas construidas con el término de clase natural "esmeralda" son inválidas y las construidas con "esmefiro" son válidas. Más bien, es un caso en el cual las inferencias que contienen el término "esmeralda" son inductivamente válidas, pero conllevan conclusiones falsas, dadas las extrañas travesuras del mundo. Y 
esto no tiene por qué sorprender, pues las inducciones válidas no siempre son preservadoras de verdad.

\section{El trasfondo "fregeano" y la definición ostensiva}

Lo que debemos examinar ahora es si esta forma de enfrentar el nuevo enigma de la inducción es concluyente. En especial, debemos concentrarnos en la cuestión de si la teoría de la referencia de Putnam no supone una apelación tácita al sistema de creencias del hablante para fijar la extensión de los términos de clase natural. Si esta apelación existe, entonces no sólo la teoría de Putnam no resultaría tan externalista como en ocasiones se ha pretendido, sino que, además, la respuesta recién esbozada al nuevo enigma de la inducción se debilitaría. Esto se debe a que parte de la fuerza de esta respuesta radica, precisamente, en la idea de que los términos de clase natural contienen un elemento indexical en el cual las creencias de los hablantes no desempeñan ningún papel. Así, si encontramos que la fijación de la extensión de los términos de clase natural está mediada de algún modo por el sistema de creencias del hablante, esta mediación podría llevar a que comunidades lingüísticas con creencias diferentes fijaran la extensión de sus términos de clase natural de formas diferentes $\mathrm{y}$, de este modo, la validez de las inferencias inductivas podría verse relativizada a los diferentes lenguajes, tal y como Goodman pretende.

Podemos comenzar prestando atención a la siguiente historia que nos cuenta Goodman:

La Sra. de Oropel analizó un muestrario en su tapicería y, tras hacer su selección, encargó suficiente género como para tapizar silla y sillón, insistiendo en que el género encargado debería ser exactamente igual que la muestra. Cuando recibió el paquete, lo abrió ansiosamente y creyó morir cuando cayeron al suelo revoloteando cientos de pequeñas piezas rectangulares, de cinco por ocho centímetros, con los bordes cuidadosamente recortados en zig-zag, exactamente como los de la muestra. Cuando llamó a la tienda, protestando enérgicamente, el dueño le contestó dolido y amargado: "Pero, Sra. de Oropel, Ud. insistió en que el género debería ser exactamente igual a la muestra. Cuando llegó ayer de 
fábrica, puse a todos los dependientes hasta altas horas de la noche a cortarlo para que fuera igual que esa muestra, como Ud. quería."

El incidente casi había sido olvidado meses después cuando la señora de Oropel, que había cosido entre sí todas las piezas y había tapizado sus sofás, decidió dar una fiesta. Fue a su pastelería y, tras seleccionar un pastel de chocolate de entre los que había en el mostrador, encargó que le enviaran, dos semanas más tarde, suficiente pastel como para sus cincuenta invitados. Cuando la gente empezaba a llegar a la fiesta, llegó también un camión que portaba un inmenso pastel de chocolate. La dama, que corrió consternada a la pastelería al verlo, quedó desarmada ante la queja de la pastelera: "No se puede imaginar, Sra. de Oropel, el problema que tuvimos. Mi marido, que es el encargado de la tapicería, me advirtió que su encargo debería realizarse en una sola pieza." (Goodman 1990, pp. 94-95)

Salta a la vista que en esta historia tanto la desafortunada señora de Oropel como el tapicero se encuentran ante la misma muestra de tela. Algo similar ocurre con el caso del pastel de chocolate. El origen del malentendido radica, sin embargo, en que el pedacito de tela y el pastel del mostrador no ejemplifican la misma cantidad de propiedades para los protagonistas del relato. Mientras que a la señora de Oropel le parece que la muestra de tela ha de ejemplificar únicamente el diseño que ha de exhibir la tela con la que pretende tapizar sus muebles, el tapicero entiende que el tamaño del pedazo de tela también es relevante. En el caso del pastel de chocolate ocurre justamente lo contrario. A la señora de Oropel le parece que el tamaño del pastel de muestra debe tenerse en cuenta al preparar su encargo, mientras que la panadera - dadas las advertencias de su esposo- no lo considera relevante. La moraleja de esta historia entonces salta a la vista: una muestra ejemplifica solamente ciertas propiedades, aun cuando tenga mucha más. Conviene notar, además, que la razón de los malentendidos entre la señora de Oropel y sus interlocutores no radica en las muestras, sino en que cada uno considera que ellas ejemplifican propiedades diferentes. Incluso podrían pensarse casos en los que las propiedades de unas muestras seleccionadas por cada uno de los protagonistas de la historia no coincidieran en absoluto. 
Casos como éstos llevan a Goodman a preguntarse qué es lo que hace que una muestra sea válida o, para decirlo en otros términos, que ejemplifique ciertas propiedades y no otras. Una muestra no puede ejemplificar todo el conjunto de propiedades que posee, pues en tal caso ella sería una muestra únicamente de sí misma. Goodman insiste en que la muestra tampoco ejemplifica sus propiedades "intrínsecas", pues las características que se ejemplifican varían según las circunstancias y las prácticas adoptadas. Así, en el caso de la muestra del tapicero, el tamaño no debe ser ejemplificado, mientras que en el caso del pastel sí. Incluso Goodman advierte que una misma muestra puede ejemplificar propiedades diferentes en contextos diferentes:

Aunque una muestra textil normalmente enseña la textura, etc., y no la forma o el tamaño si se muestra como contestación a la pregunta "¿qué es una muestra de tapicero?", funcionará no como una muestra del género sino como una muestra de lo que es una muestra de tapicero, de forma que el tamaño y la forma estarán ahora entre las cualidades de las que es ejemplo. (Goodman 1990, pp. 95-96)

De acuerdo con esto, una muestra no puede ser válida o inválida per se, o ejemplificar en sí misma y en todos los casos las mismas propiedades. Por el contrario, la validez de la muestra o el tipo de propiedades que ejemplifica dependerá de que "se conforme con toda una práctica adecuada de interpretación de muestras y ejemplos" (Goodman 1990, p. 182).

Debe notarse que esta respuesta guarda un estrecho parecido con la respuesta de Goodman al nuevo enigma de la inducción. Allí tampoco tenía sentido preguntar cuándo un predicado es proyectable en sí mismo o una inferencia inductiva es válida per se, sino que más bien la respuesta dependía del grado de atrincheramiento que el predicado o el tipo de inferencia inductiva tenían al interior de una práctica lingüística. De hecho, la pregunta acerca de la validez de una muestra es en muchos sentidos similar a la pregunta acerca de la validez de una inferencia inductiva. Cuando nos preguntamos qué características ejemplifica una muestra, en última instancia nos estamos preguntando 
si una cierta característica es proyectable a toda una clase de objetos. Incluso puede pensarse que el pasaje citado al comienzo de esta sección constituye, mutatis mutandis, una formulación del nuevo enigma de la inducción aplicada al caso de las muestras. En ambos casos, encontramos un cuerpo de evidencia compatible con varias generalizaciones, ninguna de ellas determinada en sí misma por dicha evidencia. De la misma forma que en un caso nos deja estupefactos el que alguien proyecte la hipótesis de que todas las esmeraldas son verdules a partir de la evidencia disponible, en el otro nos sorprende que el tapicero proyecte "tamaño" ante un pedacito de tela cuando se trata de tapizar unos muebles. Vistas así las cosas, resulta curioso que concedamos tan fácilmente que la validez de una muestra varía según las circunstancias y las prácticas asociadas y, sin embargo, nos resistamos a pensar que lo mismo puede ocurrir con la validez de una inferencia inductiva.

Llegados a este punto podemos retomar la preocupación acerca de si en la teoría de Putnam existe alguna apelación tácita al sistema de creencias de los hablantes para fijar la extensión de los términos de clase natural. De entrada, llama la atención el hecho de que en el acto mismo de fijar la extensión - tal y como Putnam lo entiende - exista una relación muestra/clase natural: señalamos una muestra (un vaso de agua, por ejemplo) y decimos que es agua si y sólo si está en la relación mismo $L$ con lo que llamamos "agua", o también decimos que agua es todo aquello que está en la relación mismo ${ }_{L}$ con esta muestra de agua. Lo que debemos preguntarnos ahora es si este caso es similar al caso de la señora de Oropel, o si existe alguna diferencia esencial entre ellos. Si encontráramos que no hay ninguna diferencia esencial entre ambas situaciones, las consideraciones de Goodman con respecto a la validez de las muestras y la proyectabilidad podrían aplicarse al nivel mismo de la fijación de la extensión y, de este modo, la respuesta al nuevo enigma de la inducción esbozada en la sección anterior estaría condenada al fracaso.

Si queremos evitar que esto ocurra, la diferencia esencial entre el caso de la fijación de la extensión de "agua" y el caso del pedacito de tela ha de radicar en lo siguiente: en el caso de 
"agua" debe evitarse que ante la misma muestra diferentes comunidades fijen de forma distinta la extensión de "agua", dados sus diferentes sistemas de creencias. En el caso del pedacito de tela, el problema radica justamente en una interferencia desafortunada de creencias distintas. La señora de Oropel cree que la muestra del tapicero forma parte de la clase tela cuadriculada, pero no está incluida en la clase tela de tamaño adecuado para forrar sofás. En cambio, el tapicero —en virtud de algún malentendido - cree que la muestra de tela forma parte de esta última clase.

Con todo, la teoría de la referencia de Putnam — tal y como está formulada en "El Significado de "Significado" — no logra impedir que comunidades con distintos sistemas de creencias fijen de forma diferente la extensión de los términos de clase natural. Hay en dicha teoría un trasfondo "fregeano" oculto. La razón de ello radica en que el término "líquido" de la relación "mismo $L "$ forma parte del marcador semántico del significado de "agua", es decir, la creencia de que el agua es un líquido constituye una creencia que difícilmente estaríamos dispuestos a abandonar acerca del agua. Pero si es así, ¡nuestras creencias sobre el agua desempeñan un papel relevante en la fijación de la extensión del término "agua"! Justamente, la relación mismo $L$ y, a fortiori, el elemento "líquido", son fundamentales para la determinación de la extensión de "agua". Sin ellos, no podríamos garantizar que este término designa rígidamente, ni podríamos fijar su extensión más allá del señalamiento de una muestra de agua dada. De esta manera, la teoría de Putnam supone una apelación tácita al conjunto de creencias e intereses de los hablantes para la fijación misma de la extensión. Lo curioso del caso es que el propio Putnam concede en "El Significado de "Significado"" que la relación mismo $L$ es teórica y luego afirma:

Para que algo sea agua, por ejemplo, ha de guardar la relación mismo $_{L}$ con ciertas cosas. Pero ¿qué relación es mismo $L$ ? $x$ está con $y$ en la relación mismo $L$ sólo en el caso de que (1) $x$ e $y$ sean los dos líquidos, y (2) $x$ e $y$ compartan importantes propiedades físicas. El mismo término "líquido" es una palabra que designa una clase natural que no intentaré analizar aquí. El término "propiedad" es un término de amplio espectro que hemos analizado en 
otros escritos. Quiero detenerme ahora en la noción de importancia. La importancia es una noción relativa a intereses. (Putnam 1991, p. 157)

Así pues, parece que la teoría de Putnam está mucho más cerca de las que defienden sus adversarios de lo que algunos filósofos consideran. Esto se debe a que si el paso implícito por el sistema de creencias es inevitable para fijar la extensión de un término de clase natural mediante la definición ostensiva, entonces habremos de aceptar la idea fregeana de que la intensión de un término determina su extensión en un sentido mucho menos trivial que en el que Putnam afirma que la extensión constituye un elemento del vector del significado. De esta forma, puede decirse que si bien Putnam tuvo razón cuando se quejó de que las teorías tradicionales de la referencia olvidaban la contribución de la sociedad y el mundo al momento de fijar la extensión de un término, a él se le olvidaron los compromisos teóricos y las creencias de los hablantes; en una palabra, las intensiones.

Ahora bien, el que el trasfondo "fregeano" presente en la teoría de Putnam desempeñe un papel importante en la fijación de la extensión de los términos de clase natural pone de relieve una importante suposición en la argumentación de Putnam. Él construye el caso de las dos Tierras para mostrar que dos hablantes o comunidades lingüísticas distintas pueden mantener sistemas de creencias idénticos y, sin embargo, sus términos pueden poseer extensiones diferentes. A partir de allí, él concluye que, contrario a lo que se había pensado tradicionalmente, las creencias del hablante no desempeñan ningún papel importante en la determinación de la referencia. Sin embargo, esta conclusión no se deriva por sí sola de la argumentación de Putnam. Para alcanzarla, él tendría que garantizar, además, que no hay ninguna mediación importante de las creencias en la fijación de la extensión de un término de clase natural, de tal modo que dos términos podrían tener idéntica extensión aun cuando las creencias asociadas por los hablantes a cada uno de ellos fueran totalmente diferentes. El problema es que el caso de las dos Tierras no puede mostrar esto de ningún modo, pues precisamente está construido sobre la base de que los hablantes de las 
dos Tierras tienen exactamente las mismas creencias acerca del líquido que llaman "agua".

De esta manera, los ejemplos de Putnam a lo sumo prueban que las creencias de los hablantes subdeterminan la extensión de sus términos. Esto ciertamente puede ser útil para cuestionar una teoría de la referencia en la que se sostenga que las creencias determinan totalmente la referencia. Con todo, no por ello debe olvidarse el importante papel que cumplen las creencias de los hablantes en la fijación de la referencia. Precisamente, cuando Putnam reconoce que nuestros sistemas de creencias subdeterminan la extensión de nuestros términos, su teoría de la referencia se transforma de forma significativa. Por ello, en obras posteriores a "El Significado de "Significado" ", él insiste en que si el mundo fija la referencia de nuestros términos de clase natural, siempre lo hace en función de nuestros compromisos teóricos particulares o, para decirlo con una de sus metáforas, "la mente y el mundo construyen conjuntamente la mente y el mundo" (Putnam 1988, p. 13). De acuerdo con esto, puede pensarse que una de las razones que llevó a Putnam a emprender una revisión sustancial de sus antiguas tesis filosóficas y a rechazar el realismo metafísico en nombre del así llamado realismo interno radica, justamente, en el reconocimiento de la esencial dependencia de la referencia con respecto a nuestros sistemas de creencias.

Desafortunadamente, el reconocimiento de este trasfondo "fregeano" presente en la teoría de la referencia de Putnam debilita una respuesta realista al nuevo enigma de la inducción, tal y como la que se esbozó en la sección anterior. Precisamente, gran parte de la fuerza de dicha respuesta radicaba en la idea de que los términos de clase natural poseen un elemento indexical oculto en el cual los sistemas de creencias de una comunidad dada no desempeñan ningún papel. Pero si ahora se reconoce que tales creencias sí tienen un papel relevante en la fijación de la extensión de los términos de clase natural, entonces comunidades con creencias e intereses diferentes podrían fijar la extensión de sus términos de formas distintas y, de esta manera, la validez de las inferencias inductivas construidas con ellos se vería relativizada a las diferentes prácticas lingüísticas, tal y como sugiere Goodman. Para ilustrar esto, puede imaginarse un 
extraño pueblo chamánico en el cual —dados sus particulares intereses y creencias - se utilicen definiciones ostensivas como " "verdul' es todo aquello que está en la relación mismo color que esta esmeralda" (y lo que cuenta para ellos como mismo color que no es lo que cuenta para nosotros como tal), para que los excéntricos predicados goodmanescos resulten proyectables en tal comunidad. Pero ni siquiera necesitamos un caso tan artificial, sino que podemos ilustrar el mismo punto con el siguiente comentario de C.Z. Elgin con respecto al ejemplo de las dos Tierras:

Para decidir si se debe incluir una muestra particular de un líquido en la extensión de "agua", lo que es importante para un químico es su composición química; para un biólogo, su capacidad para sustentar la vida; y para un barman el que sea potable cuando se mezcla con whisky. Así, la pregunta "¿se debe incluir XYZ en la extensión de "agua?" bien puede contestarse con otra pregunta: "¿quién quiere saberlo?" (Elgin 1983, p. 37)

De este modo, mientras que la comunidad de químicos podría fijar la extensión de "agua" a través de la relación misma estructura molecular y, de este modo, excluir XYZ; los barmen podrían preferir apelar a la relación misma potabilidad al mezclarse con whisky, de forma que XYZ podría formar parte de la extensión de "agua". Y en ambos casos, tanto la manera como se proyectó el término "agua" como el tipo de inducciones que se considerarían válidas sería diferente en virtud de los diferentes intereses y prácticas lingüísticas de cada comunidad. Es más, aún si se le concede al realista robusto que el mundo en sí mismo está constituido por ciertas clases reales, puede ocurrir que ellas no correspondan con las clases que se consideran legítimas al interior de una práctica lingüística dada, tal y como sucede en el caso de los barmen. ${ }^{3}$

3 Incluso C.Z. Elgin (1995) ha argumentado que las presuntas clases que conformarían la estructura última del mundo no tienen que coincidir necesariamente con las clases que la ciencia considera genuinas en tanto que el interés de los científicos por descubrir las categorías últimas de la realidad bien puede estar subordinado a la búsqueda de ciertas virtudes epistémicas que se consideren más valiosas en las teorías. Con todo, no voy a examinar la argumentación de Elgin en este ensayo. 
Con todo, la principal razón por la cual se invalida el intento de enfrentar el nuevo enigma de la inducción apelando a la teoría de la referencia de Putnam radica en que el trasfondo "fregeano" que la alimenta pone de manifiesto que ella, por sí misma, deja intacto el problema de la proyectabilidad. Esto se debe a que el paso que se da de la muestra actual a toda la clase que ella ejemplifica a través de la definición ostensiva involucra en sí mismo dicho problema. Si, por ejemplo, definimos ostensivamente "caballo" como todo aquello que está en la relación ser lo mismo que esta muestra caballuna, aún tenemos que decidir qué cuenta y qué no cuenta como lo mismo en este contexto, esto es, aún tenemos que decidir cómo vamos a proyectar el término "caballo" para que cubra otros objetos fuera de la muestra dada y excluya a otros. De esta forma, el trasfondo "fregeano" de la teoría de Putnam sirve para mostrar no sólo que siempre tenemos que decidir acerca de la manera como vamos a proyectar un término para cubrir nuevas instancias, sino también que dicha decisión depende esencialmente de nuestros propios intereses y sistemas categoriales. En este sentido, podemos cerrar esta sección con un pasaje de Razón, verdad e historia que tiene un talante claramente goodmaniano:

Debemos observar en este punto que "del mismo tipo" es una expresión que no tiene sentido si no es desde un sistema categorial que señale qué propiedades cuentan y qué propiedades no cuentan como semejanzas. Después de todo, cualquier cosa es del mismo tipo que cualquier otra de varias maneras. (Putnam 1988, p. 62)

\section{Propiedades intrínsecas y nominalismo: el fondo de la cuestión}

Parte de la argumentación de la sección anterior estaba sustentada en la concepción de Goodman acerca de las muestras. Precisamente, el reconocimiento del trasfondo fregeano del que se nutre la teoría de la referencia de Putnam se basó en el hecho de que el acto mismo de fijación de la extensión involucra una relación muestra/clase en la cual el sistema de creencias de una comunidad cumple un papel fundamental. Ahora bien, el que 
los sistemas categoriales desempeñen un papel definitivo incluso en el ámbito de las muestras y la definición ostensiva lleva a que Goodman, y el mismo Putnam en un momento posterior de su desarrollo filosófico, sostenga que una muestra dada no es válida o inválida per se ni ejemplifica en sí misma determinadas propiedades y no otras. En este sentido, no es extraño que, al final del pasaje que acabo de citar, Putnam —en un espíritu muy goodmaniano - declare que "cualquier cosa es del mismo tipo que cualquier otra de varias maneras". Sin embargo, debe notarse que en este contexto esta afirmación resulta sintomática: con ella se pone de relieve el trasfondo nominalista que alimenta la concepción de Goodman con respecto a las muestras y a la proyectabilidad en general. Lo que debemos preguntarnos entonces es si esta apelación tácita al nominalismo no invalida el argumento de la sección anterior. La razón de ello radica en que ahora el realista robusto podría argumentar que en este contexto una apelación al nominalismo constituye una petición de principio, pues lo que está en cuestión aquí es, precisamente, si tiene sentido decir que las cosas guardan entre sí ciertas semejanzas intrínsecas ajenas a todo sistema de creencias, o más bien sólo cabe decir — como sugiere el nominalista goodmaniano- que las semejanzas que atribuimos a los objetos sólo tienen sentido dentro de una práctica lingüística específica. Sin duda, este reclamo del realista robusto resulta justo. Con él, se pone de manifiesto que la discusión en torno al nuevo enigma de la inducción no sólo debe darse en los niveles semántico y epistémico, sino que también involucra la clásica disputa ontológica entre esencialismo y nominalismo.

Desde esta perspectiva, el realista robusto podría sostener que si bien su solución al nuevo enigma de la inducción se ve debilitada por el trasfondo fregeano que alimenta la teoría de Putnam, esto no significa que dicha solución sea del todo indefendible. Para mostrar esto, el realista robusto argumentaría que, cuando el nominalista afirma que la semejanza sólo tiene sentido al interior de un marco categorial, pasa por alto que el hecho mismo de que dos objetos resulten semejantes bajo un esquema dado es sólo un hecho relacional que presupone un hecho más básico acerca de los objetos en consideración: el 
hecho de que tales objetos poseen en sí mismos propiedades intrínsecas. Para decirlo en palabras de Mackie:

Una sola cosa, por sí misma, tiene características o propiedades, y podemos elegir sus propiedades intrínsecas como aquellas que (lógicamente) tendría, independientemente de que hubiera o no otras cosas. Con respecto a estas propiedades intrínsecas, la cosa es de este u otro tipo haya o no haya otras cosas que formen estos "tipos" junto con ella. Si cada una de dos cosas tiene, así, por sí misma, cierta propiedad, entonces es una consecuencia lógica que se asemejarán entre sí en este sentido. Y dos cosas no pueden tener la relación de semejanza exacta en cierto sentido excepto como una consecuencia lógica de que cada una de ellas tenga, por sí misma, cierta característica. La realidad fundamental es de hecho aquella sobre la cual la posición aristotélica llama la atención: el hecho de que cada cosa tiene cualidades en sí misma, su ser como es en varias maneras. [...] Cualquier análisis reductivo concebible de esta realidad fundamental no evitará una circularidad: tendremos que postular casos de esta forma: cosa-intrínsecamente-de-ciertotipo, entre los datos de cualquier explicación que intentemos dar. (Mackie 1988, pp. 167-168)

Esta argumentación le permite sostener al realista robusto que si bien es cierto que en ocasiones las semejanzas que atribuimos a los objetos dependen de nuestras prácticas lingüísticas, también es cierto que dos objetos serán semejantes en sí mismos cuando compartan ciertas propiedades intrínsecas que no dependen en absoluto ni de nuestras prácticas vigentes de clasificación ni de nuestros sistemas categoriales. De acuerdo con esto, el realista robusto podría rehabilitar su respuesta al nuevo enigma de la inducción apelando a una teoría estrictamente directa de la referencia basada en la noción misma de propiedad intrínseca. De acuerdo con esta teoría, podríamos fijar la extensión de un término de clase natural como "agua" de la siguiente forma: esto es agua si y solamente si en sí mismo posee las propiedades intrínsecas (cualesquiera que sean) de lo que llamamos "agua". La ventaja de la teoría estrictamente directa de la referencia sobre la teoría de Putnam radica entonces en que las propiedades intrínsecas que comparten los objetos no dependerán en absoluto de nuestros intereses o sistemas de creencias y, de esta forma, 
no habrá lugar aquí para el trasfondo "fregeano". Así las cosas, el realista robusto podría sostener que la proyectabilidad de un término de clase natural no dependerá de nuestras prácticas vigentes, sino que más bien estará dictada por la naturaleza misma de los objetos: Un término será proyectable si y solamente si tiene como extensión un conjunto de objetos que en sí mismos comparten idénticas propiedades intrínsecas (cualesquiera que sean).

No obstante, esta propuesta, tal y como está formulada, resulta insuficiente. Aun cuando la noción de propiedad intrínseca fuera suficientemente clara o se pudiera precisar sin incurrir en un círculo vicioso, ${ }^{4}$ la teoría estrictamente directa de la referencia debe complementarse con una teoría epistemológica acerca del acceso epistémico directo a las propiedades intrínsecas de los objetos. De lo contrario, el esfuerzo del realista robusto por defender su salida al nuevo enigma de la inducción podría verse amenazado por casos en los cuales términos con diferentes creencias asociadas podrían compartir la misma extensión, o por casos en los cuales ocurre exactamente lo contrario. Un ejemplo del primer caso sucedería si habitáramos un mundo que es goodmanesco en el siguiente sentido: las propiedades intrínsecas comunes a los objetos no son propiedades como ser verde o ser esmeralda, sino que los objetos en sí mismos poseen propiedades intrínsecas goodmanescas como ser verdul o ser esmefiro. Desde esta perspectiva, queda claro que cuando definiéramos ostensivamente "esmeralda" como todo aquello que comparte las mismas propiedades intrínsecas que esta esmeralda, ex hypothesi, la extensión del término "esmeralda" no serían las esmeraldas tradicionales sino los esmefiros, independientemente de cuáles sean nuestras creencias asociadas al término "esmeralda". Los términos "esmeralda" y "esmefiro" serían entonces coextensivos y, de este modo, la extensión de los términos de un pueblo con creencias goodmanescas sería la misma que la de nuestros términos actuales, aun cuando las creencias asocia-

4. Quine considera las dificultades que presenta la noción de clase natural en Quine 1986. Estas dificultades se presentan también, mutatis mutandis, con respecto a la noción de propiedad intrínseca. 
das a tales términos en cada caso fuesen distintas. Pero el caso contrario también puede presentarse: Supongamos que existen dos Tierras, una de ellas es goodmanesca y la otra no. Si ahora suponemos que los hablantes de ambas Tierras poseen términos con idénticas creencias asociadas, entonces estaremos ante un caso en el cual tales términos poseerán el mismo contenido de creencias, pero tendrán una extensión distinta.

Casos como éstos ponen de relieve que una teoría estrictamente directa de la referencia que no se complemente con una tesis epistemológica sobre el acceso epistémico a las propiedades intrínsecas de los objetos puede conllevar a que la extensión de un término resulte radicalmente diferente de lo que creemos. Esto no sólo traería como consecuencia el que la extensión real de nuestros términos de clase natural en última instancia nos resultara inescrutable o incognoscible (después de todo, la evidencia disponible hasta ahora no nos permite saber si nuestro universo es goodmanesco o no), sino que además pondría en entredicho el intento del realista robusto de resolver el nuevo enigma de la inducción en términos de una teoría estrictamente directa de la referencia. Precisamente, si la proyectabilidad de un término queda garantizada por el solo hecho de que, al definirlo ostensivamente, apelemos a las propiedades intrínsecas de los objetos (cualesquiera que sean) que constituyen su extensión, entonces, estrictamente hablando, todos los términos definidos ostensivamente serían proyectables. El término "esmefiro" sería tan proyectable como "esmeralda" en tanto que su extensión, bien sean esmeraldas o esmefiros, se fijaría ostensivamente apelando únicamente a las propiedades intrínsecas (cualesquiera que sean) de los objetos en cuestión y las creencias que asociemos a ambos términos serían completamente irrelevantes. Pero una solución al nuevo enigma de la inducción en la que tanto "esmeralda" como "esmefiro" resulten proyectables no es satisfactoria, pues el reto consiste en mostrar que sólo uno de ellos lo es.

La cuestión entonces es si se puede proporcionar una teoría epistemológica que complemente la teoría estrictamente directa de la referencia, sin tener que recaer en los problemas de la sección anterior. Y aquí la dificultad radica en que, en el contexto de una teoría epistemológica, el trasfondo fregeano parece 
inevitable. Esto se debe a que uno de los datos que cualquier teoría epistemológica debe tener en cuenta a la hora de explicar nuestra relación cognitiva con los objetos es el importante papel que desempeñan nuestros sistemas de creencias e intereses en dicha relación. En otras palabras, cualquier teoría epistemológica debe reconocer que cualquier aproximación cognitiva al mundo depende necesariamente de nuestra propia perspectiva. Incluso si el lenguaje teórico de la ciencia proporciona un conocimiento de la estructura última de los objetos en términos de su constitución molecular, ello no nos exime de decidir qué propiedades de los objetos hemos de considerar relevantes en función de nuestros intereses y esquemas categoriales. Y esto vale tanto en el nivel macroscópico como en el microscópico. Somos nosotros quienes decidimos usar el número de protones y no el número de neutrones como base para la clasificación de los diferentes elementos. Y si bien esta decisión no es de ningún modo arbitraria y está justificada teóricamente, sigue siendo nuestra decisión. Por consiguiente, el punto básico en el que insiste el nominalista es que aun cuando podamos conocer la estructura última de los objetos, este conocimiento siempre estará impregnado de nuestros sistemas categoriales y nuestros intereses. Este carácter perspectivista del conocimiento constituye —a mi juicio - la verdad epistemológica envuelta en el nominalismo de Goodman y en sus consideraciones sobre la relatividad conceptual.

Ahora bien, el que los esquemas categoriales determinen nuestra relación cognitiva con el mundo pone de manifiesto que la respuesta del realista robusto al nuevo enigma de la inducción no puede evitar la siguiente encrucijada: o bien el realista robusto tendrá que apoyarse únicamente en la teoría estrictamente directa de la referencia y, en este sentido, no podrá evitar la inaceptable idea de que, estrictamente hablando, todo término de clase natural definido ostensivamente es por ello mismo proyectable; o bien el realista robusto deberá complementar su respuesta al nuevo enigma de la inducción con una tesis epistemológica sobre nuestro conocimiento de las presuntas propiedades intrínsecas de los objetos y, de esta forma, tendrá que aceptar las razones epistemológicas que llevan a Goodman a afirmar que la proyectabilidad, al menos en un sentido importante, depende de 
nuestros intereses y prácticas lingüísticas. Con todo, debe reconocerse también que el intento del realista robusto por ofrecer su propia respuesta al nuevo enigma de la inducción permite extraer por lo menos dos moralejas con respecto al nominalismo goodmaniano. Por un lado, la respuesta epistemológica de Goodman al nuevo enigma de la inducción en sí misma no puede ser un argumento a favor de un nominalismo ontológico en el cual se afirma que no cabe pensar que los objetos en sí mismos tienen semejanzas intrínsecas. Antes, por el contrario, un nominalismo de este tipo sólo será defendible en la medida en que se ponga en entredicho la afirmación del realista robusto según la cual es un hecho fundamental que cada cosa tiene cualidades en sí misma. Por el otro $-\mathrm{y}$ en íntima conexión con lo anteriorel realista robusto pone de manifiesto que Goodman no puede inferir la tesis irrealista acerca de las clases naturales a partir de sus tesis epistemológicas sobre la proyectabilidad.

Recapitulemos ahora, a manera de conclusión, los resultados obtenidos en este ensayo. Al comienzo de este escrito expresé la esperanza de que un estudio conjunto del nuevo enigma de la inducción y las teorías directas de la referencia pudiese arrojar luces sobre ciertas dificultades presentes en cada uno de ellos. Si la línea de argumentación desarrollada aquí es correcta, entonces tenemos los siguientes resultados: en primer lugar, el nuevo enigma de la inducción muestra que la teoría de la referencia directa de Putnam se nutre de un trasfondo fregeano que hace que la extensión de los términos de clase natural dependa en un sentido importante de las creencias del hablante y la comunidad lingüística. Justamente, este trasfondo fregeano es fundamental a la hora de fijar la extensión de un término de clase natural mediante una definición ostensiva. Por otro lado, se puso de manifiesto que una respuesta realista al nuevo enigma de la inducción no debe basarse únicamente en una consideración sobre el comportamiento semántico de los términos de clase natural, sino que además debe complementarse con una teoría epistemológica sobre nuestra relación cognitiva con el mundo. La necesidad de esta teoría radica en que una explicación completa de la relación entre el lenguaje y el mundo debe tener en cuenta la manera en que ciertas variables epistémicas, tales como nuestros intereses 
o sistemas de creencias, contribuyen a articular dicha relación. Asimismo, debe reconocerse que si bien el nuevo enigma de la inducción está enmarcado dentro de los planteamientos nominalistas de Goodman, por sí mismo es insuficiente como argumento a favor de una determinada posición ontológica. Es más, el nuevo enigma de la inducción constituye un problema tanto para esencialistas como para nominalistas y, en este sentido, es independiente de nuestras simpatías ontológicas. Esto se debe a que, bien sea que las clases naturales constituyan la estructura última del mundo o dependan del lenguaje, la fijación misma de la extensión de los términos de clase natural supone enfrentar el problema de la proyectabilidad. Siempre debemos determinar cómo vamos a proyectar un término más allá de una muestra y ello se hará en función de nuestros intereses y compromisos teóricos. El resultado general de este ensayo puede entenderse entonces como un intento de mostrar la necesidad de que una teoría sobre la fijación de la extensión de los términos de clase natural se integre adecuadamente con una teoría epistemológica acerca de la manera en que construimos y evaluamos nuestras inciertas inferencias inductivas.

\section{BIBLIOGRAFÍA}

Devitt, M., 1991, Realism and Truth, 2a. ed., Princeton University Press, Princeton, Nueva Jersey.

Elgin, C.Z., 1995, "Unnatural Science”, Journal of Philosophy, XCII, 6, pp. 289-302.

—_, 1983, With Reference to Reference, Hackett, Indianápolis/Cambridge.

Goodman, N., 1995, De la mente y otras materias, Visor, Madrid.

— 1990, Maneras de hacer mundos, Visor, Madrid.

__, 1983, Fact, Fiction and Forecast, 4a. ed., Harvard University Press, Cambridge, Mass.

Lewis, D., 1997, "New Work for a Theory of Universals", en C.Z. Elgin (comp.), Nelson Goodman's New Riddle of Induction, Garland, Nueva York/Londres, pp. 175-210.

Mackie, J.L., 1988, Problemas en torno a Locke, trad. Adriana Sandoval, Instituto de Investigaciones Filosóficas-unam, México. 
EL NUEVO ENIGMA DE LA INDUCCIÓN Y LOS TÉRMINOS DE CLASE NATURAL 85

Putnam, H., 1991, "El Significado de "Significado"”, en Luis Ml. Valdés (comp.), La búsqueda del significado, Tecnos, Madrid, pp. 131-193.

, 1988, Razón, verdad e historia, Tecnos, Madrid.

Quine, W.V.O., 1986, "Géneros naturales", en La relatividad ontológica y otros ensayos, trad. M. Garrido, Tecnos, Madrid, pp. 147176.

Recibido el 12 de junio de 2001; aceptado el 7 de noviembre de 2001 